\title{
Drell-Yan and vector boson plus jets measurements with the ATLAS detector
}

\author{
Markus Zinser*, on behalf of the ATLAS Collaboration \\ Institut für Physik, Johannes Gutenberg-Universität Mainz \\ E-mail: markus.zinser@cern.ch
}

\begin{abstract}
The inclusive production of $\mathrm{W}$ and $\mathrm{Z}$ bosons as well as the off-shell $\mathrm{Z} /$ gamma production are standard candles at hadron colliders. The measurement of their production cross-sections can be compared to theory calculations at NNLO QCD and has an impact on our knowledge of the parton densities of the proton. Run-1 studies carried out by the ATLAS Collaboration are reviewed and first LHC Run-2 results are included. Measurements of the transverse momentum of Z/gamma bosons have been performed in different di-lepton invariant mass and rapidity regions. These measurements are sensitive to soft resummation effects and hard jet emissions for small and large momentum transfers, respectively, probing QCD in a unique way. Productions of light and heavy-flavour jets in association with a $\mathrm{W}$ or a $\mathrm{Z}$ boson in proton-proton collisions are important processes to study QCD in multi-scale environments and have sensitivity to parton density functions. The ratio of $(\mathrm{Z}+\mathrm{jets}) /(\mathrm{W}+\mathrm{jets})$ provides a precise test of $\mathrm{QCD}$ due to the large cancellations of theoretical and experimental uncertainties.
\end{abstract}

The European Physical Society Conference on High Energy Physics

22-29 July 2015

Vienna, Austria

\footnotetext{
* Speaker.
} 


\section{Introduction}

ATLAS [1] has performed several measurements of vector boson production both inclusively and with associated light or heavy flavour jets at the Large Hadron Collider (LHC). W- and Zbosons are produced abundantly at the LHC, thanks to their large production cross section. The leptonic decay of $W$ - and $Z$-bosons provides a clean experimental signature. These decays can be used to probe higher-order quantum-chromo-dynamics (QCD) effects as well as constrain parton distribution functions (PDFs) and to measure electroweak (EWK) parameters. These measurements are reaching high precision and allow for detailed comparison with theoretical predictions. The precise cross section measurements presented here are based on the full data set collected by the ATLAS detector in the year 2011 of proton-proton collisions at a center of mass energy of $\sqrt{s}=7 \mathrm{TeV}$. All measurements are done separately for the electron and the muon channel, corrected to a common phase space region and then combined.

\section{2. $W, Z$ plus jets measurements}

\section{1 $W+$ jets measurement}

The $W+$ jets production cross section has been measured up to 7 additional jets using the 2011 data set with an integrated luminosity of $4.6 \mathrm{fb}^{-1}$ [2]. The jets are clustered using the anti- $k_{\mathrm{T}}$ [3] algorithm with a radius parameter $R=0.4$. The measurement has been carried out in a fiducial volume which closely relates to the selection at detector level. Jets with transverse momenta starting from $30 \mathrm{GeV}$ up to $1 \mathrm{TeV}$ have been included in a large rapidity acceptance region $|y|<4.4^{1}$. At these high transverse momenta higher-order EWK effects can become as important as those from perturbative QCD ( $\mathrm{pQCD}$ ) corrections. The cross section has been measured inclusively and differentially in several observables and can be a valuable input for Monte Carlo tuning. Figure 1 shows the cross section for the production of $\mathrm{W}+\mathrm{jets}$ as a function of the inclusive jet multiplicity. The measured cross section is compared to a number of theoretical predictions at both LO and NLO in PQCD. The leading-order predictions are using ALPGEN [4], SHERPA [5, 6] and HEJ [7, 8]. The next-to-leading order predictions are obtained using MEPS@NLO [5, 6] and BLACKHAT+SHERPA $[9,10,11]$. All agree within the assigned experimental uncertainties. ALPGEN and SHERPA show some opposite trend towards higher jet multiplicities.

\section{2 $W+$ jets/Z+jets ratio measurement}

When comparing the $W+$ jets and $Z+$ jets [12] cross section measurements, the ratio $R_{\text {jets }}=$ $\sigma(W+j e t s) / \sigma(Z+j e t s)$ between these two is a much more precise test of pQCD, since several experimental uncertainties and effects from non-perturbative processes, such as hadronization and multi-parton interactions, are greatly reduced in the ratio. The cross section has been measured inclusively and differentially in several observables [13]. Figure 2 shows on the left side the $R_{\text {jets }}$

\footnotetext{
${ }^{1}$ ATLAS uses a right-handed coordinate system with its origin at the nominal interaction point (IP) in the centre of the detector and the $z$-axis along the beam pipe. The $x$-axis points from the IP to the centre of the LHC ring, and the $y$-axis points upward. Cylindrical coordinates $(r, \phi)$ are used in the transverse plane, $\phi$ being the azimuthal angle around the beam pipe. The pseudorapidity is defined in terms of the polar angle $\theta$ as $\eta=-\ln \tan (\theta / 2)$.
} 

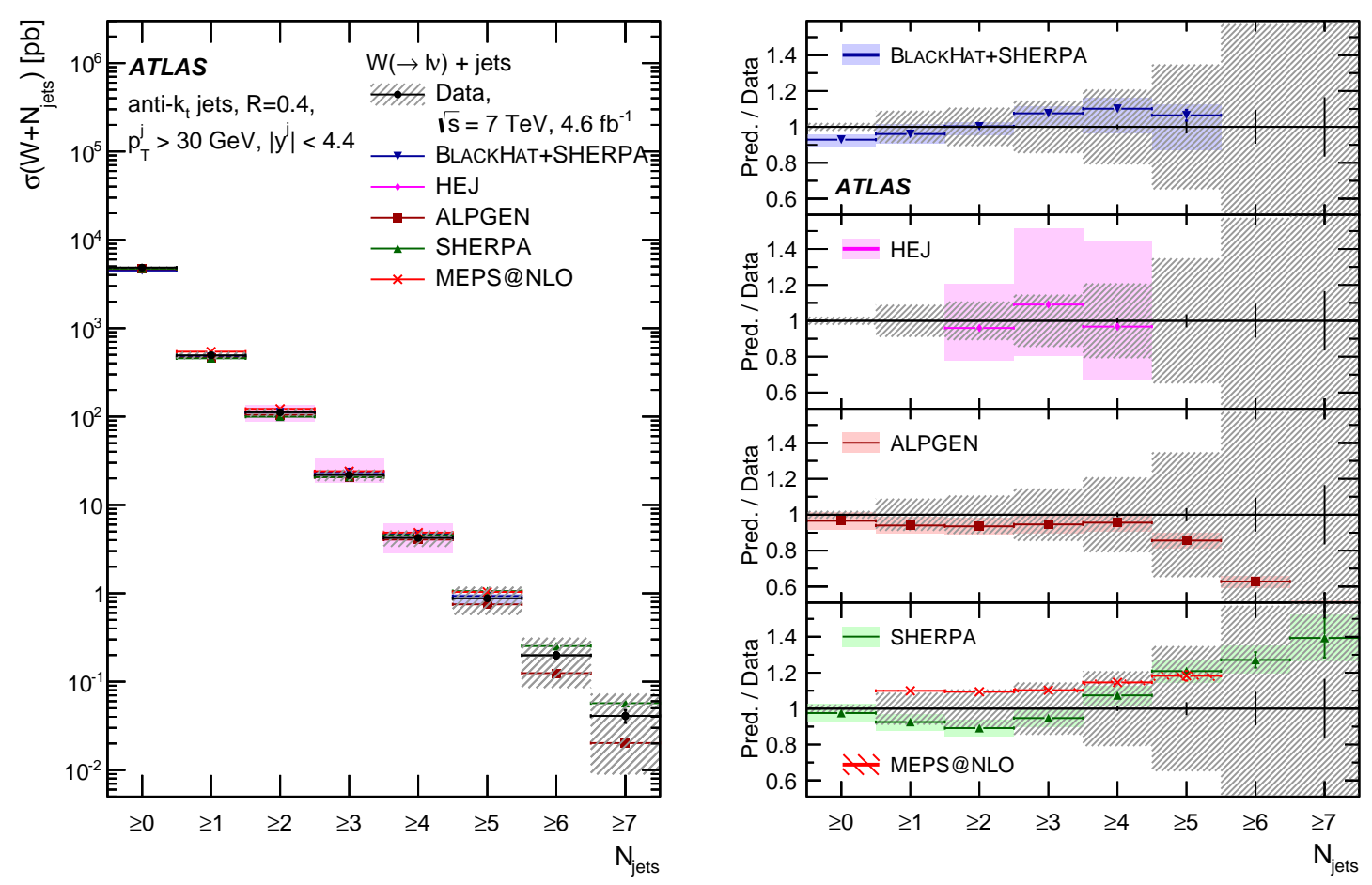

Figure 1: Cross section for the production of $\mathrm{W}+\mathrm{jets}$ as a function of the inclusive jet multiplicity [2]. The measured cross section is compared to predictions from BLACKHAT+SHERPA, HEJ, ALPGEN, SHERPA and MEPS@ NLO. The left-hand plot shows the differential cross section and the right-hand plot shows the ratios of the predictions to the data.

measurement as a function of the inclusive jet multiplicity. The ratio is compared to a prediction calculated with BLACKHAT+SHERPA and the prediction of the ALPGEN and SHERPA generators. Small deviations can be seen at larger $N_{j e t}$ for the comparison with SHERPA.

\section{$2.3 Z+b-j e t s$ measurement}

ATLAS has measured the fully unfolded particle-level cross section for the production of $Z$ bosons in association with one or two $b$-jets [14]. This is a process for which an improved understanding is becoming important at the LHC, since it forms a major background to several new physics searches, and in particular to the search for $H \rightarrow b \bar{b}$ in associated production with a $W$ or $Z$. The cross section has been measured inclusively and differentially in several observables for the one and two $b$-jet final state. Figure 2 shows on the right side the measured cross section as function of the angular separation of the two $b$-jets $\Delta R(b, b)$. The measurement is compared to the NLO predictions from MCFM [15] and AMC@ NLO [16] both using the MSTW2008 [17] PDF set. Also overlaid are LO multi-legged predictions for ALPGEN+HERWIG+JIMMY [18, 19] and SHERPA. None of the predictions is able to describe the data at low angular separation, which is the region where the data is sensitive to gluon splitting. 

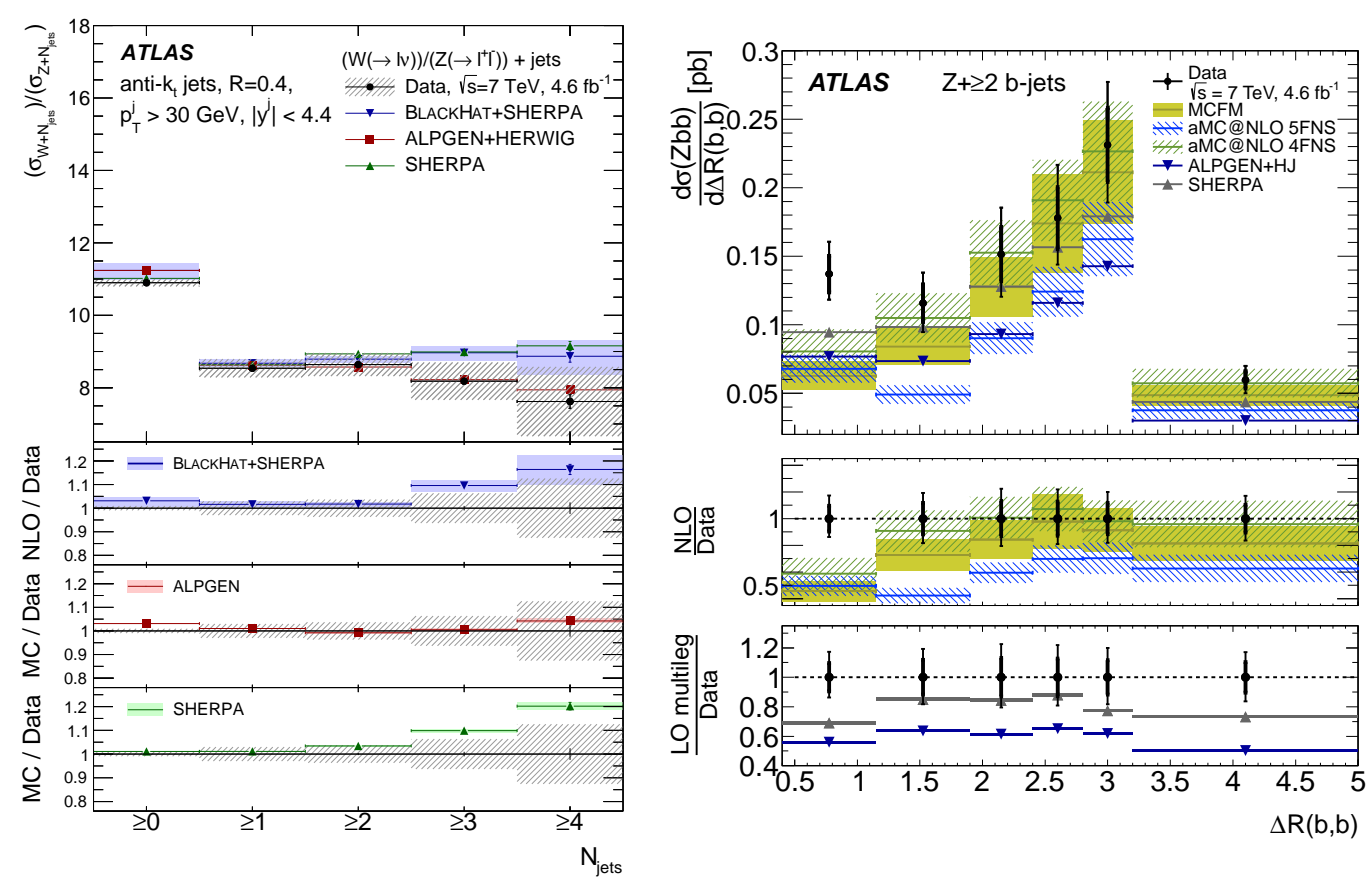

Figure 2: Left: Ratio of $W+$ jets and $Z+$ jets cross section, $R_{\text {jets }}$, as a function of inclusive jet multiplicity [13]. Ratios of the BLACKHAT+SHERPA NLO calculations and the ALPGEN and SHERPA generators to the data are shown in the lower panels. Right: The cross section $\sigma(Z b b)$ as a function of $\Delta R(b, b)$ [14]. The top panel shows the measured differential cross-sections. Overlayed for comparison are the NLO predictions from MCFM and AMC@NLO both using the MSTW2008 PDF set. Also overlaid are LO multi-legged predictions for ALPGEN+HERWIG+JIMMY and SHERPA. The middle panels show the ratio of NLO predictions to data, and the lower panels show the ratio of LO predictions to data.

\section{3. $Z-p_{\mathrm{T}}$ measurement}

Measurements of boosted $Z$-boson production, i.e. with non-zero $p_{\mathrm{T}}$, can be used to constrain theoretical models of $\mathrm{pQCD}$. The low $Z-p_{\mathrm{T}}$ region can probe resummation techniques whereas the high $Z$-boson $p_{\mathrm{T}}$ regime probes higher-order $\mathrm{QCD}$ corrections. The measurement is an important input to constrain the parton shower parameters of Monte Carlo generators. A correct modeling of the $Z-p_{\mathrm{T}}$ (as well as $W-p_{\mathrm{T}}$ ) spectrum is important for many physics analyses, e.g. the $\mathrm{W}$ mass measurement. ATLAS performed a measurement inclusively up to $Z$ absolute rapidities of 2.4 as well as in three rapidity bins [20]. Figure 3 shows on the left side the ratio of the measured $Z$ - $p_{\mathrm{T}}$ cross section with predictions from FEWZ [21, 22, 23] and DYNNLO [24, 25]. Both do not include any resummation effects which results in a large discrepancy at low $p_{\mathrm{T}}$. On the right side the measurement is compared to RESBOS $[26,27]$ which is in agreement within the estimated uncertainties at low $p_{\mathrm{T}}$, as it includes these resummation effects. All three predictions show a discrepancy at around $40 \mathrm{GeV}$ in $Z-p_{\mathrm{T}}$.

\section{First Run-II $W, Z$ results}

After a two year long shut down, the ATLAS started to take data from proton-proton colli- 

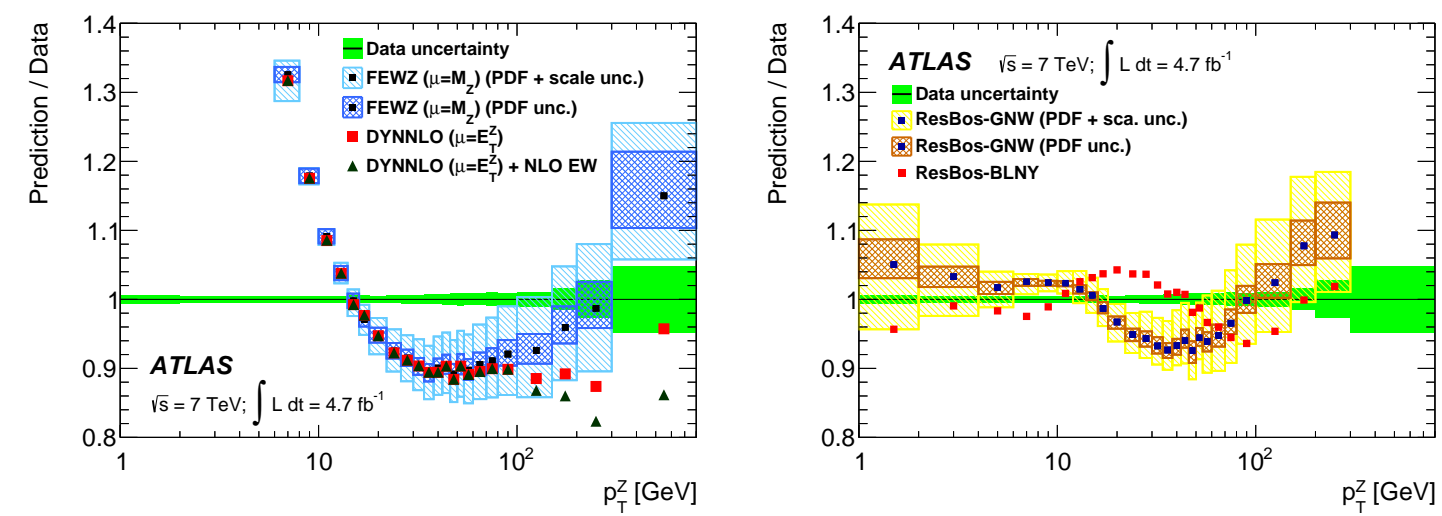

Figure 3: Ratio between the combined Born-level $Z-p_{\mathrm{T}}$ measurement and predictions by different computations: FEWZ, DYNNLO (left) and REsBos (right) [20].

sions at a center of mass energy of $\sqrt{s}=13 \mathrm{TeV}$. The study of leptonic $W$ and $Z$ boson decays is ideal for validating the early detector performance, such as lepton reconstruction, and the analysis software chain. Figure 4 shows on the left side the dilepton mass distribution after a $Z \rightarrow e^{+} e^{-}$ selection [28]. On the right side the transverse mass distribution calculated from the lepton and the missing transverse energy ( $E_{\mathrm{T}}^{\text {miss }}$ ) after a $W \rightarrow \mu v$ selection is shown. The data for both selections are in good agreement with the expectation. The systematic uncertainties shown are obtained using very early studies as well as extrapolation from the Run-I uncertainties.
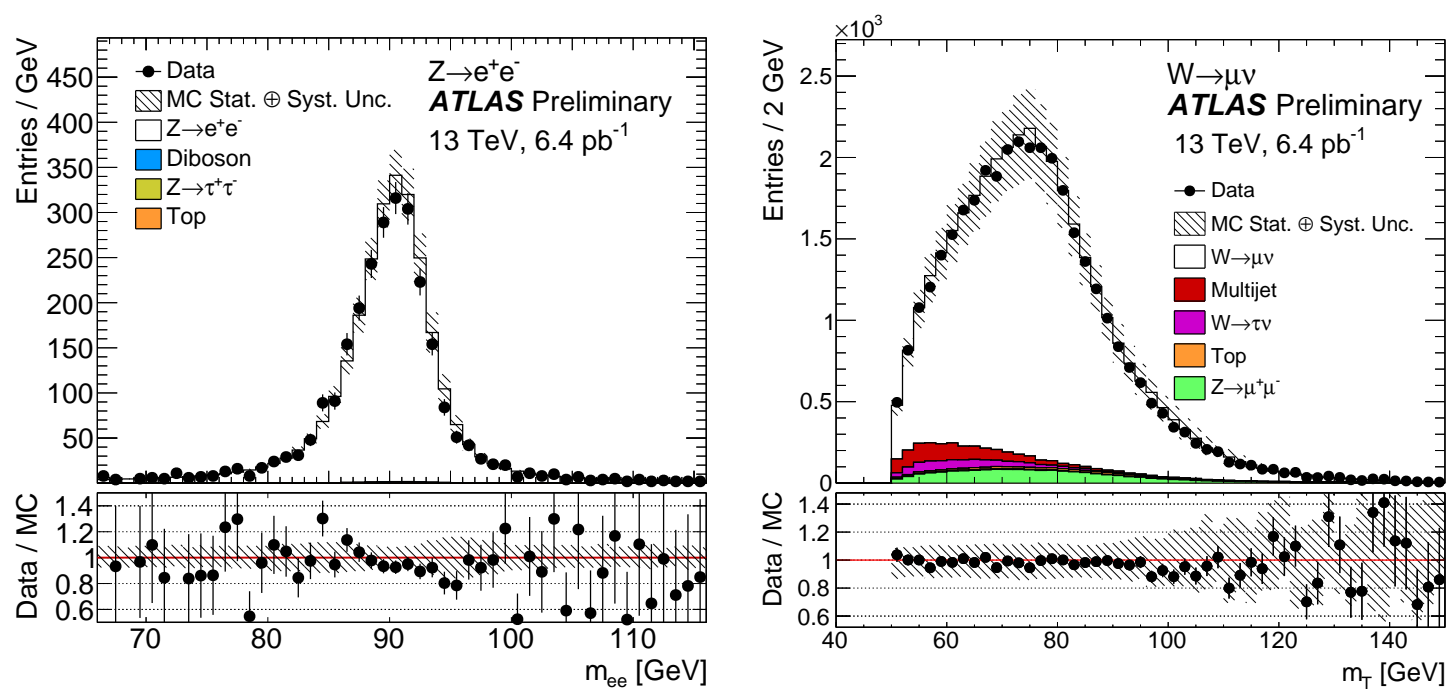

Figure 4: Dilepton mass distribution after the $Z \rightarrow e^{+} e^{-}$selection (left) and transverse mass distribution calculated from the lepton and the $E_{\mathrm{T}}^{\mathrm{miss}}$ after $W \rightarrow \mu v$ selection (right). The data are compared to the expected contributions from all backgrounds [28]. The luminosity uncertainty, which is not shown, is $9 \%$.

\section{Conclusion}

The analyses presented here cover a range of precision measurements of $W$ and $Z$ production 
at ATLAS in proton-proton collisions ranging from a center of mass energy from $7 \mathrm{TeV}$ to $13 \mathrm{TeV}$. All $7 \mathrm{TeV}$ precision analyses show overall a good agreement with the theory predictions. First studies of the $13 \mathrm{TeV}$ data show a good understanding of the early ATLAS detector performance and are agreeing with the theoretical expectation.

\section{References}

[1] ATLAS Collaboration, JINST 3 (2008) S08003.

[2] ATLAS Collaboration, G. Aad et al., Eur. Phys. J. C75 no. 2, (2015) 82, arXiv:1409. 8639 [hep-ex].

[3] M. Cacciari, G. P. Salam, and G. Soyez, JHEP 04 (2008) 063, arXiv:0802.1189 [hep-ph] .

[4] M. L. Mangano, M. Moretti, F. Piccinini, R. Pittau, and A. D. Polosa, JHEP 07 (2003) 001, arXiv:hep-ph/0206293 [hep-ph].

[5] T. Gleisberg, S. Hoeche, F. Krauss, M. Schonherr, S. Schumann, F. Siegert, and J. Winter, JHEP 02 (2009) 007, arXiv:0811.4622 [hep-ph].

[6] S. Hoeche, F. Krauss, M. Schonherr, and F. Siegert, JHEP 04 (2013) 027, arXiv: 1207.5030 [hep-ph].

[7] J. R. Andersen, T. Hapola, and J. M. Smillie, JHEP 09 (2012) 047, arXiv:1206. 6763 [hep-ph].

[8] J. R. Andersen and J. M. Smillie, JHEP 01 (2010) 039, arXiv:0908.2786 [hep-ph] .

[9] Z. Bern, L. J. Dixon, F. Febres Cordero, S. HÃúche, H. Ita, D. A. Kosower, D. MaÃôttre, and K. J. Ozeren, Phys. Rev. D88 no. 1, (2013) 014025, arXiv:1304.1253 [hep-ph] .

[10] C. F. Berger, Z. Bern, L. J. Dixon, F. Febres Cordero, D. Forde, T. Gleisberg, H. Ita, D. A. Kosower, and D. Maitre, Phys. Rev. D80 (2009) 074036, arXiv: 0907.1984 [hep-ph].

[11] C. F. Berger, Z. Bern, L. J. Dixon, F. Febres Cordero, D. Forde, T. Gleisberg, H. Ita, D. A. Kosower, and D. Maitre, Phys. Rev. Lett. 106 (2011) 092001, arXiv: 1009.2338 [hep-ph] .

[12] ATLAS Collaboration, G. Aad et al., JHEP 07 (2013) 032, arXiv:1304. 7098 [hep-ex] .

[13] ATLAS Collaboration, G. Aad et al., Eur. Phys. J. C74 no. 12, (2014) 3168, arXiv: 1408.6510 [hep-ex].

[14] ATLAS Collaboration, G. Aad et al., JHEP 10 (2014) 141, arXiv:1407.3643 [hep-ex] .

[15] J. M. Campbell and R. K. Ellis, Nucl. Phys. Proc. Suppl. 205-206 (2010) 10-15, arXiv:1007.3492 [hep-ph].

[16] J. Alwall, R. Frederix, S. Frixione, V. Hirschi, F. Maltoni, O. Mattelaer, H. S. Shao, T. Stelzer, P. Torrielli, and M. Zaro, JHEP 07 (2014) 079, arXiv:1405.0301 [hep-ph] .

[17] A. D. Martin, W. J. Stirling, R. S. Thorne, and G. Watt, Eur. Phys. J. C63 (2009) 189-285, arXiv:0901.0002 [hep-ph].

[18] G. Corcella, I. G. Knowles, G. Marchesini, S. Moretti, K. Odagiri, P. Richardson, M. H. Seymour, and B. R. Webber, JHEP 01 (2001) 010, arXiv: hep-ph/0011363 [hep-ph] .

[19] J. M. Butterworth, J. R. Forshaw, and M. H. Seymour, Z. Phys. C72 (1996) 637-646, arXiv:hep-ph/9601371 [hep-ph]. 
[20] ATLAS Collaboration, G. Aad et al., JHEP 09 (2014) 145, arXiv:1406.3660 [hep-ex] .

[21] K. Melnikov and F. Petriello, Phys. Rev. D74 (2006) 114017, arXiv: hep-ph/ 0609070 [hep-ph].

[22] R. Gavin, Y. Li, F. Petriello, and S. Quackenbush, Comput. Phys. Commun. 182 (2011) 2388-2403, arXiv:1011.3540 [hep-ph].

[23] Y. Li and F. Petriello, Phys. Rev. D86 (2012) 094034, arXiv:1208.5967 [hep-ph].

[24] S. Catani, L. Cieri, G. Ferrera, D. de Florian, and M. Grazzini, Phys. Rev. Lett. 103 (2009) 082001, arXiv:0903.2120 [hep-ph].

[25] S. Catani and M. Grazzini, Phys. Rev. Lett. 98 (2007) 222002, arXiv: hep-ph/ 0703012 [hep-ph].

[26] C. Balazs and C. P. Yuan, Phys. Rev. D56 (1997) 5558-5583, arXiv: hep-ph/ 9704258 [hep-ph].

[27] M. Guzzi, P. M. Nadolsky, and B. Wang, Phys. Rev. D90 no. 1, (2014) 014030, arXiv:1309.1393 [hep-ph].

[28] ATLAS Collaboration, ATL-PHYS-PUB-2015-021, 2015, https://cds.cern.ch/record/2037696. 